\title{
ESC-BRAIN: Experimental and Clinical Stroke Research - Do They Connect?
}

\section{Meeting Report of the ESC-BRAIN Joint Symposium Held in London and Shanghai in May 2013}

\author{
J.-C. Baron ${ }^{a} \quad$ I.M. Macrae ${ }^{\text {b }}$ H.P. Adams Jr. ${ }^{c} \quad$ U. Dirnagl ${ }^{d}$ \\ a Department of Neurology, Inserm U894, Centre Hospitalier Sainte Anne, Sorbonne Paris Cité, Paris, France; \\ ${ }^{b}$ Wellcome Surgical Institute \& Glasgow Experimental MRI Centre, Institute of Neuroscience and Psychology, \\ College of Medical, Veterinary and Life Sciences, University of Glasgow, Glasgow, UK; 'Division of Cerebrovascular \\ Diseases, Department of Neurology, Carver College of Medicine, Comprehensive Stroke Center, University of \\ lowa Hospitals and Clinics, University of lowa, lowa City, lowa, USA; ${ }^{d}$ Center for Stroke Research Berlin, Charité \\ Universitätsmedizin Berlin, and German Center for Neurodegeneration Research, Berlin, Germany
}

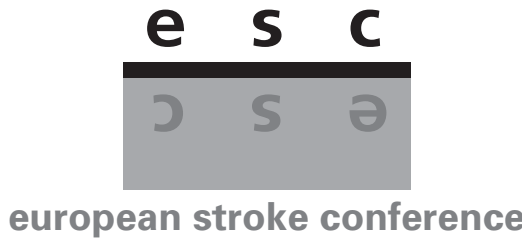

\section{Introduction}

At the European Stroke Conference in London this year, a special ESC-BRAIN Joint Symposium was held and presented by the same speakers both in London and Shanghai (2 weeks earlier) at the BRAIN 2013 Conference (alias the International Symposium on Cerebral Blood Flow, Metabolism and Function). The aim of this symposium, which was convened by M. Hennerici, U. Dirnagl and J.C. Baron, was to address the burning question of the blatant discrepancy between the positive effects of neuroprotectants in reducing infarct size and sometimes also improving functional outcome in animal models of stroke, and the failure of neuroprotection in man in randomized trials so far [1]. It was hoped that by convening experts from both the preclinical and clinical fields in the same symposium and presenting the same talks in front of more basic and clinically oriented audiences, a better dialogue

\section{KARGER}

(c) 2013 S. Karger AG, Basel

1015-9770/13/0364-0306\$38.00/0

E-Mail karger@karger.com

www.karger.com/ced
BRAIN 2013

Shanghai

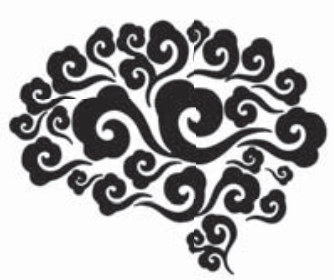

导言

今年在伦敦举行的欧洲卒中大会期间, 大会的讲 演人先后在伦敦和上海 (此前两周) 安排并出席了 ESC（欧洲卒中大会）和BRAIN2013（又名 “国际脑 血流与脑代谢论坛” ) 共同举办的研讨会。这个由 M. Hennerici, U. Dirnag1和J. C. Baron召集的研讨会 旨在探讨神经保护剂在缩小梗死面积、改善卒中动 物模型预后中的积极作用，及其在人体随机对照试 验中的不尽如人意的表现之间的这种明显的矛盾。 本次研讨会把临床前专家和临床专家邀请到一起, 在从事更为基础和临床研究导向的听众前探讨相同 的议题，希望籍此在不同类别的专家间展开更好的 对话, 也许还能就上述矛盾给出合理的解释。

\footnotetext{
Jean-Claude Baron, MD, ScD, FMedSci

Inserm U894

2 ter rue d'Alésia

FR-75014 Paris (France)

E-Mail jean-claude.baron@inserm.fr

Prof. Dr. Ulrich Dirnagl

Department of Neurology and Experimental Neurology and

Center for Stroke Research Berlin, Charité Universitätsmedizin Berlin

Charitéplatz 1, DE-10117 Berlin (Germany)

E-Mail ulrich.dirnagl@charite.de
} 
between these communities would ensue, and perhaps explanations for these discrepancies would emerge.

Two specific issues were identified as both particularly controversial and potential sources of discrepancies, namely the duration of salvageable tissue in animals versus man, and the outcome measures used to assess the effects of interventions. The following four articles by the speakers of this symposium provide a brief but to-thepoint overview of the state of the art regarding these two issues in both the experimental and clinical domains. They highlight similarities but also some differences between the findings and approaches, and propose pragmatic ways to better connect and effectively bridge the gap so that future preclinical studies assessing interventions, gain from the scientific rigor of modern clinical trials. For example, inclusion of randomization, blinding, systematic follow-up or powered large samples [1-3]. Conversely, improvements could be made in the design of future clinical trials to reproduce the specific experimental conditions under which an agent has shown benefit in preclinical tests, e.g. in combination with or following reperfusion, in anterior circulation strokes $[1,4,5]$.

Bearing these major, frustrating discrepancies in mind, strong connections do exist between preclinical and clinical stroke, such as the ischemic penumbra and the benefit from intravenous tissue plasminogen activator treatment, which were first documented in animal models [6, 7] and subsequently proven in man, to name only two discoveries that have revolutionized stroke care.

It was clear from the positive way this symposium was received at both conferences that both communities are determined to, and actually already engaged in, the advancement and strengthening of this connection, which beyond any doubt will eventually effectively improve the outcome of stroke victims.

\section{J.C. Baron, U. Dirnagl, M. Hennerici}

有两个议题被认为是出现上述矛盾的特别有争议 的及潜在的原因, 即动物和人各自的救治时间窗, 及评估介入预后的方法。下面这四篇由研讨会的讲 演人撰写的文章，言简意赅地提供了这两个议题在 实验和临床领域中的最新进展, 强调了在研究结果 和研究方法中的相同点和不同点, 提出了实用的方 法以更好更有效地处理两者间的差异。希望未来的 临床前研究达到诸如随机对照实验、盲法实验、系 统评价或大样本试验等现代临床试验的严谨性所带 来的介入水平。而将来的临床试验设计方案亦能有 所改进, 能再现某种药物在临床前试验中发挥功效 的特定实验条件，如在前循环卒中的治疗中配合使 用再灌注法。

虽然存在着这些重要的、令人沮丧的不一致，但 临床前和临床卒中研究仍有着紧密的联系, 仅以对 缺血半暗带的研究和静脉注射t-PA疗法为例, 这两 项让卒中治疗发生彻底变化的成果, 都是先在动物 模型中取得成功, 然后在人体治疗中得到证实的。

通过这次研讨会，两类专家决定像已经开始的那 样，继续发展和强化彼此的联系。而这毫无疑问将 会改善卒中患者的预后水平。

\section{References}

1 Fisher M: New approaches to neuroprotective drug development. Stroke 2011;42:S24-S27.

-2 Fisher M, Feuerstein G, Howells DW, Hurn PD, Kent TA, Savitz SI, Lo EH: Update of the Stroke Therapy Academic Industry Roundtable preclinical recommendations. Stroke 2009;40:2244-2250.

-3 Dirnagl U, Hakim A, Macleod M, Fisher M, Howells D, Alan SM, Steinberg G, Planas A, Boltze J, Savitz S, Iadecola C, Meairs S: A concerted appeal for international cooperation in preclinical stroke research. Stroke 2013;44:1754-1760.

4 Muir KW: Heterogeneity of stroke pathophysiology and neuroprotective clinical trial design. Stroke 2002;33:1545-1550.
5 Saver JL, Albers GW, Dunn B, Johnston KC, Fisher M: Stroke Therapy Academic Industry Roundtable (STAIR) recommendations for extended window acute stroke therapy trials. Stroke 2009;40:2594-2600.

6 Astrup J, Siesjo BK, Symon L: Thresholds in cerebral ischemia - the ischemic penumbra. Stroke 1981;12:723-725.

7 Zivin JA, Fisher M, DeGirolami U, Hemenway CC, Stashak JA: Tissue plasminogen activator reduces neurological damage after cerebral embolism. Science 1985;230:1289-1292. 


\section{Time Is Brain: What Do We Know about the Time Window for Reperfusion and Neuroprotection in Rodents?}

I. Mhairi Macrae

Wellcome Surgical Institute \& Glasgow Experimental MRI Centre, Institute of Neuroscience and Psychology, College of Medical,

Veterinary and Life Sciences, University of Glasgow, Glasgow, UK

Reviews and more recent meta-analyses of the experimental stroke literature highlight the numerous drugs which induce significant neuroprotection in rodent stroke models, none of which (with the exception of tissue plasminogen activator) have translated to man. Therefore, it is valid to pose the question that framed this year's Joint ESC and Brain 2013 Symposium: 'Experimental \& Clinical Stroke Research - Do They Connect?'

In exploring this question, one issue to examine is the available time window for restorative reperfusion and neuroprotection in rodents and to determine how this compares with the equivalent time window for the acute stroke patient [see companion article by Baron, pp. 311]. Knowledge of the available time window will improve the design of studies testing new therapeutic interventions and the power to predict those likely to translate into clinical practice. However, in designing these studies it is equally important that the time point at which outcome measures are assessed is late enough for ischaemic damage to have fully evolved. Otherwise interventions which delay rather than stop the progression of ischaemic damage could be wrongly assessed as neuroprotective.

In this overview, I will first describe how stroke is modelled in rodents, then discuss appropriate time points for assessing outcome, and finally the available time window for reperfusion and neuroprotection in rodent stroke models. To do this, I have used information on the evolution of acute brain injury (diffusion-weighted MRI), penumbra (perfusion/diffusion mismatch in MRI) and infarct size (T2-weighted MRI and histology). Functional assessment of outcome (e.g. behavioural testing of sensorimotor deficits) is equally important in assessing new therapies for stroke and is covered in the companion article by Dirnagl, pp. 315.

\section{Rodent Stroke Models}

Stroke is studied in rodents by permanently or transiently occluding a cerebral artery which causes a severe reduction in cerebral blood flow in the affected brain tissue. The middle cerebral artery (MCA) is the most com- monly affected blood vessel in human occlusive/ischaemic stroke and is the artery most frequently targeted in rodent stroke models. A range of MCA occlusion (MCAO) models has been developed and there are strengths and limitations associated with each approach (see Macrae [1]). The earliest models exposed the MCA via a surgical craniectomy. With this approach, MCAO can be achieved: by electrocoagulation of the blood within it and destruction of the blood vessel per se; mechanically, by applying an occluding device such as a clip or ligature, or pharmacologically by applying a potent vasoconstrictor with a prolonged half-life such as endothelin-1 or a pro-coagulant causing a blood clot locally. MCAO models which do not require a large craniectomy include stereotaxic injection of endothelin-1 into parenchyma adjacent to the MCA, which results in a longer lasting vasoconstriction. Alternatively, the skull is left intact and an intravascular approach via the carotid artery is used to advance a filament or embolus to the point where it blocks the origin of the MCA (fig. 1). With the exception of electrocoagulation, where the artery is permanently occluded, methods for MCAO can be adapted to induce either maintained (permanent) or transient occlusion.

An assessment of the available time window for tissue salvage can be made by determining the time from stroke onset to maximum ischaemic damage. The evolution of ischaemic damage has been investigated in histology studies where groups of animals are killed at different time points after MCAO, or in serial imaging studies where longitudinal data are collected from each animal to determine when the infarct reaches its maximum size. In general, acute ischaemic damage matures over a longer period following transient MCAO (tMCAO, days) compared to maintained MCAO (hours).

\section{Evolving Ischaemic Damage: Histological Approach}

Ischaemic tissue evolves over time to form the infarct, which can be identified by histology and is defined by neuropathologists as irreversible damage to all the cells within the affected parenchyma. Within the infarct, neurones show morphological features of irreversible damage (e.g. pyknosis and cytoplasmic eosinophilia on haematoxylin- and eosin-stained sections) and the neuropil is disrupted and vacuolated. Histology studies, using the permanent MCAO model in the rat and assessing ischaemic damage (corrected for brain swelling) at time points from $1 \mathrm{~h}$ to 7 days after MCAO, found ischaemic damage was maximal by $4 \mathrm{~h}$ and matures faster (maximal 


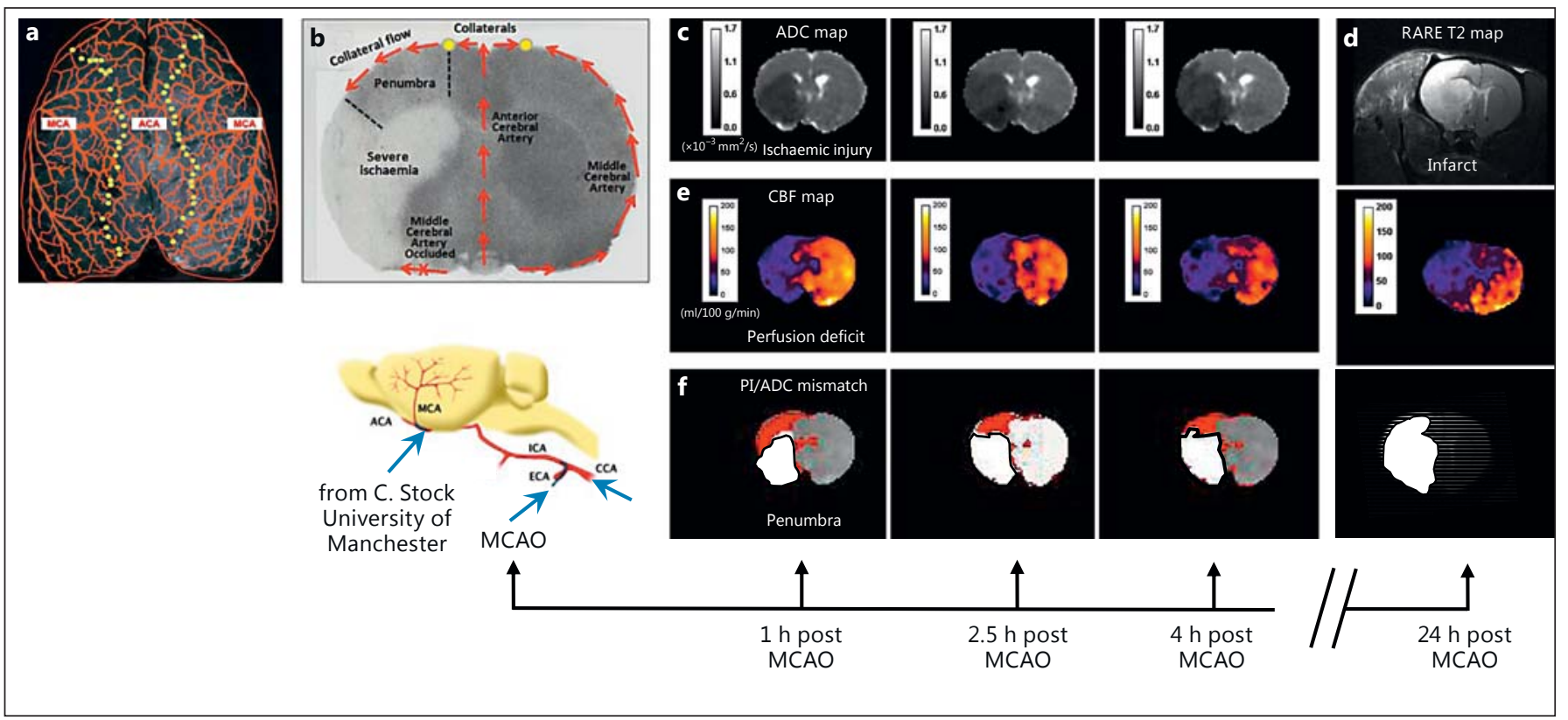

Fig. 1. Serial MRI from a rat middle cerebral occlusion (maintained MCAO) experiment. The left-hand panels illustrate the position of collateral vessels (yellow symbols in $\mathbf{a}, \mathbf{b}$ ) and the introduction of an intraluminal filament into the external carotid artery (ECA) or common carotid artery (CCA) and advanced to block the origin of the MCA. A blood flow autoradiogram (b) displays the pattern of blood flow reduction following MCAO and the position of collateral vessels which provide collateral flow from the anterior

within $2 \mathrm{~h}$ ) in the caudate nucleus than in the cortex [2, 3]. This implies that the time window for restorative reperfusion is up to $4 \mathrm{~h}$ in the rat.

\section{Evolving Ischaemic Damage: Serial Imaging Approach}

Multimodal MRI can provide serial information on the severity and location of ischaemia (perfusion imaging), acute ischaemic damage to the brain parenchyma (diffusion-weighted imaging, DWI), the cerebrovasculature (blood-brain barrier breakdown with contrast-enhanced T1) and eventual infarct (T2-weighted imaging) in each animal.

DWI, based on the brownian motion of water molecules, reveals acute ischaemic injury due to cytotoxic oedema caused by the net movement of water from the extracellular to the intracellular compartment. The restricted diffusion of water within cells results in an increased signal intensity. In the pre-clinical literature, diffusion

ESC-BRAIN: Experimental and Clinical Stroke Research cerebral artery (ACA) to support penumbral tissue. c Acute ischaemic injury (darker contrast) on ADC maps which increases over time and (d) infarct (bright contrast) at $24 \mathrm{~h}$ on T2. e The reduction in cerebral blood flow (CBF) induced by MCAO. f Penumbra (red) identified as tissue within the perfusion deficit which does not show evidence of ischaemic injury on the ADC maps (white). Penumbra decreases in size over time as ADC lesion grows. By $24 \mathrm{~h}$, no penumbra remains, having been incorporated into the infarct.

data are often presented as an image map of the apparent diffusion coefficient (ADC), where low ADC values (fig. 1c) correspond to high signal intensity on DWI scans. However, these abnormalities may signify severe ischaemia rather than irreversible ischaemic damage as the tissue can permanently return to normal diffusion if promptly reperfused.

In the rat model of permanent $\mathrm{MCAO}, \mathrm{ADC}$-defined ischaemicinjury increases in size within minutes of MCAO, reaching a plateau within 3-4 h (fig. 1c), which is approximately equivalent to the infarct measured on T2 scans at $24 \mathrm{~h}$ or later (fig. 1d). Thus, the MRI data are consistent with the histopathological data reviewed above that the time window for restorative reperfusion is up to $4 \mathrm{~h}$ in the rat.

\section{The Ischaemic Penumbra}

ADC maps can be co-registered with acute perfusion scans (fig. 1e) to identify tissue which is hypoperfused but not yet showing reduced ADC. This perfusion-diffusion 
mismatch (fig. 1f) is used as a marker of penumbra, which remains viable for a limited time (hours) after MCAO due to supplementary blood flow from the anterior and posterior cerebral arteries via collateral vessels (fig. 1a, b). Penumbral tissue is capable of recovery if promptly reperfused and in rodent embolic stroke models, tissue plasminogen activator restores flow and reduces infarct volume within a similar time frame to man [4]. In permanent MCAO models, the amount of hypoperfused tissue (perfusion deficit) remains relatively stable over the first 4-6 h, and penumbral tissue gradually becomes incorporated into the $\mathrm{ADC}$-defined lesion and final infarct (fig. $1 \mathrm{~d}, \mathrm{f})$. In rat models of maintained MCAO, the lifespan of penumbra (from perfusion-diffusion mismatch) is approximately 4-6h, again suggesting the time window for restorative reperfusion lies within this time period.

The amount of penumbral tissue available for rescue can vary across rodent strains and is influenced by the presence of co-morbidities. Bardutzky et al. [5] reported significantly larger volumes of perfusion-diffusion mismatch in male Wistar-Kyoto (WKY) compared to Sprague-Dawley rats (e.g. approximately $75 \mathrm{~mm}^{3}$ in WKY vs. $33 \mathrm{~mm}^{3}$ in Sprague-Dawley rats $90 \mathrm{~min}$ after MCAO, $\mathrm{p}<0.01)$ from 45-210 min after MCAO, at which point negligible $\left(<5 \mathrm{~mm}^{3}\right)$ penumbra was detected in SpragueDawley rats. Similar studies report smaller mismatch volumes in spontaneously hypertensive (117 vs. $66 \mathrm{~mm}^{3}$ for WKY, $\mathrm{p}<0.008,30 \mathrm{~min}$ after MCAO [6]) and spontaneously hypertensive, stroke-prone (34 vs. $43 \mathrm{~mm}^{3}$ for WKY, $\mathrm{p}>0.05,1 \mathrm{~h}$ after MCAO [7]) rat strains. The reported lifespan of mismatch tissue (4-6 h) is consistent across these rodent studies.

\section{The Time Window for Neuroprotection after Recanalization: Evolving Ischaemic Damage in tMCAO Models}

Most tMCAO studies in rodents generally employ the intraluminal filament model with durations of ischaemia of 60,90 and $120 \mathrm{~min}$ [8]. Beyond 2-3 h of tMCAO, infarct volumes match or exceed those induced by maintained MCAO and mortality is high. The time course of evolving ischaemic damage and the available time window for neuroprotection appear longer than for models of maintained MCAO but are difficult to determine due to greater variability in published data, the difficulty in differentiating between lesion growth due to oedema versus irreversible damage, and the paucity of studies that measure infarct size beyond $24 \mathrm{~h}$ after MCAO. Necrosis is the main mechanism for cell death in maintained MCAO models, but with tMCAO there can be a significant component of delayed apoptotic cell death and selective neuronal necrosis (incomplete infarction) [9], which relies on histological approaches rather than imaging for detection.

Both infarct and peri-infarct tissue becomes oedematous over the first $72 \mathrm{~h}$, which means that the formulae used to correct for brain swelling provide an estimate rather than a precise correction for infarct size. In closed skull models (e.g. intraluminal filament) producing large MCA territory strokes, brain swelling results in significant mortality beyond the 24 -hour time point. This may explain why many studies assess infarct at this time point. However, although some longitudinal studies report maximal infarct size at this time point (e.g. 90-min MCA and bilateral common carotid artery occlusion) others report further lesion growth beyond $24 \mathrm{~h}$ (e.g. from $48 \mathrm{~h}$ for 30 -min tMCAO up to 8 days for 80 -min tMCAO). The time for full infarct development may also increase as duration of MCAO decreases. For example, in the paper by Du et al. [10], the infarct did not increase in volume beyond $24 \mathrm{~h}$ with 90 -min MCAO plus common carotid artery occlusion, while with a 30-min occlusion the infarct did not become apparent until day 3 and increased further by day 14 .

In models of tMCAO, the volume of damage increases up to at least 24-48 hand, possibly, beyond. This implies that the time window for neuroprotection after recanalization lies within this time window.

\section{Effective Time Window for Neuroprotection following tMCAO}

Drugs administered after stroke onset have been shown to reduce infarct size and improve behavioural outcome in models of maintained MCAO and tMCAO. The available time window for tissue salvage can be estimated from studies where drug treatment is started at different time points following tMCAO. With very short periods of focal ischaemia (e.g. $30 \mathrm{~min}$ ), infarct volume can be significantly reduced within a time window of up to $6-9 \mathrm{~h}$ from stroke onset $[11,12]$ while with longer durations of ischaemia (90-120 min), significant neuroprotection has been reported within $4-6 \mathrm{~h}[13,14]$. Therefore, in rodent $t M C A O$ models, significant neuroprotection and reduction in infarct size is feasible within a time window of up to $9 \mathrm{~h}$ from stroke onset, depending on the duration and severity of the preceding ischaemia. 
In summary, in rodent models, the amount of penumbra available for salvage is influenced by the duration and severity of ischaemia, strain and the presence of comorbidities. Restorative reperfusion is possible within a timescale of $0-6 \mathrm{~h}$, and neuroprotection after recanalization has been demonstrated up to $9 \mathrm{~h}$. The time window for restorative reperfusion is not too dissimilar from available estimates in stroke patients and, with appropriately designed pre-clinical studies, rodent data should have translational value, and experimental and clinical stroke research should connect. However, at variance with the studies reviewed by Baron (see below) where individual patient data are considered, rodent studies so far have implemented group analyses only. Future rodent studies may address the issue of potential subgroups.

\section{References}

1 Macrae IM: Preclinical stroke research - advantages and disadvantages of the most common rodent models of focal ischaemia. $\mathrm{Br} \mathrm{J}$ Pharmacol 2011;164:1062-1078.

2 Dawson DA: An Investigation into the Pathophysiology of a New Model of Transient Focal Cerebral Ischaemia in the Rat; PhD thesis, University of Glasgow, 1994.

3 Gill R: Neuroprotective Studies of Excitatory Amino Acid Antagonists in Focal Cerebral Ischaemia in the Rat; $\mathrm{PhD}$ thesis, University of London, 1992.

4 Perel P, Roberts I, Sena E, Wheble P, Briscoe C, Sandercock P, Macleod M, Mignini LE, Jayaram P, Khan KS: Comparison of treatment effects between animal experiments and clinical trials: Systematic review. BMJ 2007;334:197.

5 Bardutzky J, Shen Q, Henninger N, Bouley J, Duong TQ, Fisher M: Differences in ischemic lesion evolution in different rat strains using diffusion and perfusion imaging. Stroke 2005;36:2000-2005.

-6 Letourneur A, Roussel S, Toutain J, Bernaudin M, Touzani O: Impact of genetic and renovascular chronic arterial hypertension on the acute spatiotemporal evolution of the ischemic penumbra: a sequential study with MRI in the rat. J Cereb Blood Flow Metab 2011;31:504-513.

7 Reid E, Graham D, Lopez-Gonzalez MR, Holmes WM, Macrae IM, McCabe C: Penumbra detection using PWI/DWI mismatch MRI in a rat stroke model with and without comorbidity: comparison of methods. J Cereb Blood Flow Metab 2012;32:1765-1777.

8 Carmichael ST: Rodent models of focal stroke: size, mechanism, and purpose. NeuroRx 2005;2:396-409.

9 Garcia JH, Liu KF, Ye ZR, Gutierrez JA: Incomplete infarct and delayed neuronal death after transient middle cerebral artery occlusion in rats. Stroke 1997;28:2303-2309, discussion 2310.

10 Du C, Hu R, Csernansky CA, Hsu CY, Choi DW: Very delayed infarction after mild focal cerebral ischemia: a role for apoptosis? J Cereb Blood Flow Metab 1996;16:195-201.

11 Wiegler K, Bonny C, Coquoz D, Hirt L: The JNK inhibitor XG-102 protects from ischemic damage with delayed intravenous administration also in the presence of recombinant tissue plasminogen activator. Cerebrovasc Dis 2008;26:360-366.

12 Fink K, Zhu J, Namura S, Shimizu-Sasamata M, Endres M, Ma J, Dalkara T, Yuan J, Moskowitz MA: Prolonged therapeutic window for ischemic brain damage caused by delayed caspase activation. J Cereb Blood Flow Metab 1998;18:1071-1076.

ESC-BRAIN: Experimental and Clinical Stroke Research
13 Belayev L, Liu Y, Zhao W, Busto R, Ginsberg MD: Human albumin therapy of acute ischemic stroke: marked neuroprotective efficacy at moderate doses and with a broad therapeutic window. Stroke 2001;32:553-560. 14 Zheng Z, Schwab S, Grau A, Berger C: Neuroprotection by early and delayed treatment of acute stroke with high dose aspirin. Brain Res 2007; 1186:275-280.

Prof. I. Mhairi Macrae

Wellcome Surgical Institute \&

Glasgow Experimental MRI Centre

Institute of Neuroscience and Psychology

College of Medical, Veterinary and Life Sciences

University of Glasgow, Garscube Estate

Glasgow G61 1QH (UK)

E-Mail Mhairi.Macrae@glasgow.ac.uk

\section{Time Is Brain: What Do We Know about the Time Window for Reperfusion and Neuroprotection in Man?}

Jean-Claude Baron

Department of Neurology, Inserm U894, Centre Hospitalier

Sainte Anne, Sorbonne Paris Cité, Paris, France

In acute stroke, the only proven target for therapy is the ischemic penumbra, i.e. the severely hypoperfused and nonfunctional but still viable tissue that can be rescued from infarction by early reperfusion. There are many additional, but as yet only putative, targets such as the neurovascular unit for prevention of hemorrhagic transformation and blood-brain barrier leakage, but also vasogenic edema, reperfusion injury, the no-reflow phenomenon and neuroinflammation. Nonacute targets are beyond the scope of this article.

Given the above, we will first address two distinct questions: (i) For how long after acute ischemic stroke in man is penumbral tissue present? (ii) What is the time window for reperfusion therapy? In the following. it is shown why these two questions are distinct although obviously related. The issue of neuroprotection is briefly addressed at the end of this overview.

\section{For How Long after Acute Ischemic Stroke in Man Is Penumbral Tissue Present?}

Although addressing this question may seem straightforward, in fact it is not so. This is because the only validated method to identify the penumbra in man so far is quantitative ${ }^{15} \mathrm{O}-\mathrm{PET}$, a technique not widely available and rather complex to implement. Furthermore, only patients eligible for PET, i.e. who can tolerate the procedure and do not have contraindications to arterial puncture,

Cerebrovasc Dis 2013;36:306-321 
such as thrombolysis or anticoagulation, have been studied so far. As a result, quantitative studies have not appeared since the mid-90s, i.e. they all date back to the prethrombolytic era. Only 6 studies have been published and only some of them provide complete data, with most providing limited clinical information. One advantage is the populations studied are probably close to generic anterior circulation stroke (ACS), as compared to recent studies using MRI, for example, which are biased towards candidates for reperfusion therapy. Summing across the 78 patients studied within $48 \mathrm{~h}$ of onset in the 5 studies that focused on the presence of high oxygen extraction fraction (OEF) as a marker of penumbra [1-5], the incidence of high OEF was nearly $100 \%$ within $6 \mathrm{~h}, \sim 80 \%$ within $12 \mathrm{~h}$, and between 25 and $100 \%>12 \mathrm{~h}$, although the latter figure was probably an overestimate due to the specific methodology used for image analysis [4].

In the Caen ${ }^{15} \mathrm{O}$-PET study of 30 ACS patients studied $5-18 \mathrm{~h}$ after stroke onset [6,7], for two reasons the pattern of cerebral blood flow versus oxygen consumption changes was considered rather than the mere presence of high OEF. Firstly, although high OEF is a necessary criterion for the penumbra, it is not a specific one as it can also be present in a severely hypometabolic core - doomed to infarction - as well as in benign oligemia - i.e. the mildly hypoperfused not-at-risk tissue, which likely resulted in an overestimated incidence of the penumbra in early studies. Second, it was considered that regardless of the presence of the penumbra, patients with a large core would be unlikely to have a good clinical outcome irrespective of the treatment, so that assessment of the potential response to reperfusion therapy rather than the mere presence or absence of penumbra was felt to be the key point. Accordingly, three PET profiles were defined, namely large core (large area of profound hypometabolism regardless of perfusion though usually associated with markedly reduced cerebral blood flow); penumbra-no large core; and no penumbra-no large core, which consistently corresponded to spontaneous recanalization with tissue hyperperfusion and preserved oxygen consumption. The validity of this classification was supported by the strong correlation with spontaneous outcome, namely all large core patients died within a few days from malignant edema or survivors had a poor outcome; all no penumbra-no large core patients had excellent or full recovery within a few days; whereas penumbra-no large core patients had highly variable outcomes, consistent with the notion that the fate of the penumbra depends on whether reperfusion subsequently occurs. Accordingly, only the latter profile would be amenable to reperfusion therapy. Although based on small numbers and hence of uncertain significance, the distribution of these three profiles across time was surprisingly even, i.e. roughly one third of patients belonged to each profile across the 5 - to 18-hour time window [7], with the latest penumbra-no large core profile seen $16 \mathrm{~h}$ after onset [8]. It was assumed that although initially all patients would belong to this profile, already within the first few hours some had progressed to the large core profile while others enjoyed early reperfusion. Interestingly, in the latter scenario, the neurological deficit outlasted tissue reperfusion, suggesting 'stunning' of the reperfused penumbra or extensive selective neuronal loss beyond the actual infarct [9]. The persistence of substantial areas of penumbra as late as $12-16 \mathrm{~h}$ after onset in some patients probably results from their perfusion being just below the penumbra threshold, allowing therefore a potential for prolonged survival [10]. Alternatively, perfusion might be unstable and oscillate across the penumbra threshold, so that tissue is not permanently penumbral. Conversely, patients who quickly evolve into the large core profile probably have very low cerebral blood flow throughout their affected tissue right from the onset, so that progression to irreversible damage is much faster. This in turn could be due to poor collateral circulation through the circle of Willis and leptomeningeal anastomoses, either constitutionally and/or due to aging and longstanding vascular risk factors such as hypertension.

Subsequent to the above PET studies, reports on the incidence of penumbra according to time since onset in unselected patients have been scarce. The Melbourne diffusion/perfusion MR study of 34 patients investigated 2.5-24 h after stroke onset reported an incidence close to $100 \%$ at $3 \mathrm{~h}, \sim 75 \%$ at $6 \mathrm{~h}$ and $\sim 45 \%$ at $24 \mathrm{~h} \mathrm{[11].} \mathrm{The} \mathrm{lat-}$ ter values appeared overestimated compared to the PET literature, which in retrospect probably reflects the lack of thresholding of the MR perfusion maps in this early study. Also, patients able to undergo MR likely represent a more selected population than patients receiving PET.

Studies reporting the incidence of penumbra over time in selected populations are also few. In EPITHET (Echoplanar Imaging Thrombolytic Evaluation Trial) which studied potential candidates for intravenous tissue plasminogen activator (t-PA) 3-6 h after onset, the incidence of penumbra using validated perfusion thresholds was 85\% [12]. In the DEFUSE (Diffusion and Perfusion Imaging Evaluation for Understanding Stroke Evolution) sample with similar characteristics, mismatch was differentiated into two subprofiles, namely the 'malignant' profile, defined by a large lesion on diffusion-weighted imaging with or without additional mismatch (corresponding to 


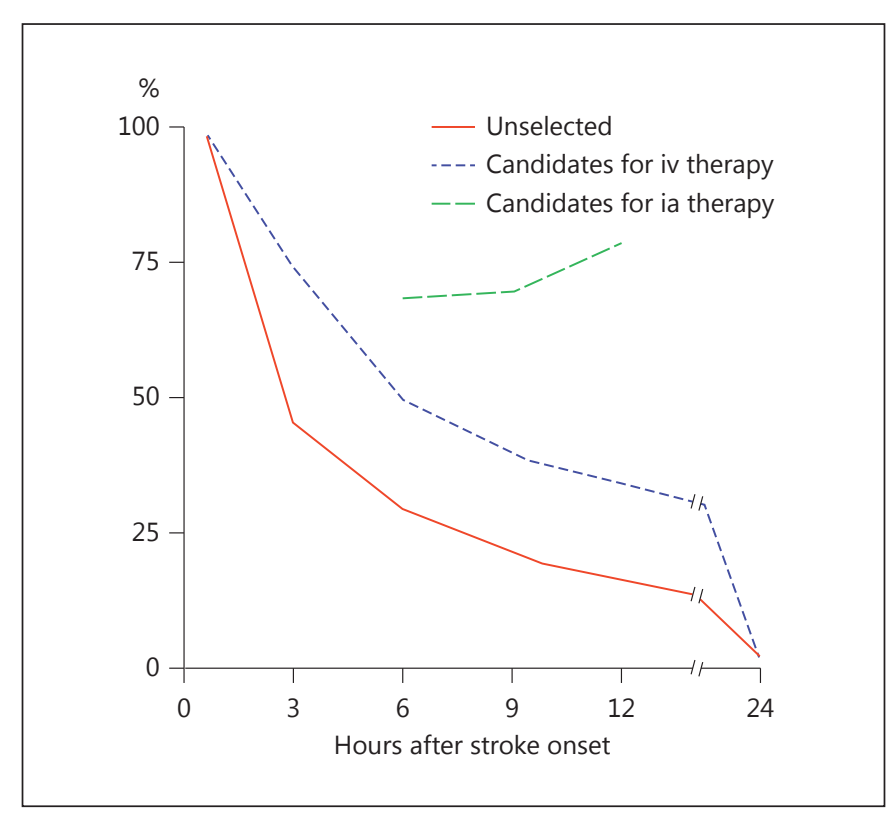

Fig. 1. Estimated incidence (\%) of the target mismatch profile according to time since clinical onset in three populations of anterior circulation stroke patients, namely unselected (red), candidates for intravenous thrombolysis (blue) and candidates for delayed intra-arterial endovascular therapy (green).

the large core PET profile above), and the 'target mismatch' profile, corresponding to the penumbra-no large core profile [13]. Using a strict definition for target mismatch, this profile was present in $51 \%$ of the DEFUSE patients and $53 \%$ of the EPITHET sample when applied to this dataset in retrospect [14]. In the DEFUSE 2 study, 138 patients were screened for potential endovascular treatment within $12 \mathrm{~h}$ of onset, including nonresponders to intravenous t-PA [15]. Target mismatch was present in 63\%. Given the open nature of this study, 34 patients did not go to the catheterization laboratory following MRI. In the remaining 104 patients judged clinically to be candidates for a late endovascular treatment, target mismatch was present in $78 \%$. Interestingly, in the latter subgroup, $68 \%$ of the patients had target mismatch within $6 \mathrm{~h}$ and $85 \%$ within 7-12 h. Thus, the incidence of target mismatch over time depends heavily on the selection bias of the sample, which remains very high even at late time points in candidates for endovascular therapy compared to candidates for intravenous therapy, and even more to unselected patients. However, in absolute terms, the number of patients eligible for endovascular therapy is very small.

Figure 1, which was constructed based on the above data, idealizes the incidence of target mismatch during the first $24 \mathrm{~h}$ after ACS onset, both for unselected and selected (intravenous and endovascular treatment) patient populations. These estimates will obviously need to be confirmed and refined. The close to $90 \%$ incidence of target mismatch within 1-2 $\mathrm{h}$ from ACS onset is consistent with the reported rate of spontaneous recanalization on conventional angiography reported by Fieschi in the 80s [16].

\section{What Is the Time Window for Reperfusion Therapy?}

As already discussed, the presence of penumbra does not necessarily predict a good response to reperfusion therapy if the core is already large. Furthermore, even target mismatch does not equate with good treatment response: first because treatments do not always result in effective and permanent recanalization, particularly with intravenous t-PA and old endovascular devices; second because recanalization inconsistently results in effective tissue reperfusion due to distal fragmented emboli or the no-reflow phenomenon; and third because the timing of effective reperfusion is critical, i.e. if it occurs after the penumbra has turned into core it is useless and may even be harmful. Finally, the presence of a core of low cerebral blood volume increases the risk of symptomatic intracerebral hemorrhage, which can negate the benefit from reperfusion. Thus, the time window for reperfusion therapy may differ from that of target mismatch.

Although trials have shown intravenous t-PA to be beneficial up to $4.5 \mathrm{~h}$ after onset, this may not just reflect reperfusion, and, given the wide confidence intervals, the benefit from true reperfusion may extend much longer. Unpublished data from the ClotBust-Pro study of transcranial Doppler-enhanced recanalization following intravenous t-PA [17] show significant benefits from recanalization still present at $5 \mathrm{~h}$ [Alexandrov et al., unpubl. data]. The PROACT II trial of intra-arterial urokinase in patients with angiographically proven middle cerebral artery stem occlusion up to $6 \mathrm{~h}$ from onset showed a significant clinical effect [18]. The recently published negative thrombectomy trials beyond $4.5 \mathrm{~h}$ go strongly against the above evidence but have been criticized on methodological grounds [19]. Meta-analyses of open studies of endovascular therapy have suggested a benefit from reperfusion up to $6 \mathrm{~h}$ [20], again with a wide confidence interval suggesting some patients benefit well beyond this limit. In another study, endovascular recanalization resulted in significantly increased good outcomes in patients with target mismatch and proximal occlusion with 
a median time of recanalization of $12 \mathrm{~h}$ (interquartile range 10-16 h) [21]. In DEFUSE 2 , tissue reperfusion resulted in a significant benefit in the target mismatch versus no-target mismatch profile, with no influence of time of therapy on this response up to $12 \mathrm{~h}$ [15]. Altogether, therefore, the available data strongly suggest benefits from reperfusion therapy in target mismatch extend up to $6 \mathrm{~h}$ with intravenous t-PA and at least up to $12 \mathrm{~h}$ with appropriate endovascular therapy. These data are consistent with the PET findings reporting the presence of penumbra-no large core (i.e. target mismatch) up to $16 \mathrm{~h}$ in a proportion of unselected patients (see above). Several randomized controlled trials using perfusion imaging selection beyond $4.5 \mathrm{~h}$ are currently ongoing to confirm or refute these data. Of note, the clinical benefit from, and potentially the time window of, delayed reperfusion therapy will be enlarged if the risk of symptomatic intracerebral hemorrhage is reduced, which may be achieved with novel thrombolytics, improved endovascular devices and perhaps addition of vascular protective therapy.

\section{What Do We Know about the Time Window for Neuroprotection in Man?}

Although a number of preclinical studies have suggested that neuroprotection applied following reperfusion may reduce final infarct size (see companion article by Macrae, pp. 308), there is no evidence towards this in man. Regarding neuroprotection applied during occlusion, the available evidence suggests that unless reperfusion takes place, neuroprotectants can only delay, but not prevent, neuronal death in the penumbra [22]. In other words, neuroprotection might extend the time window for reperfusion (i.e. 'buy time') but cannot replace reperfusion. This is supported by the failure of all trials of neuroprotectants that did not associate the agent with recanalization therapy [23]. Unfortunately, only three trials so far have used this dual design. One of them (CLASS-T) was a pilot study, another (lubeluzole) was stopped prematurely, and the zonampanel trial was never published. A trial of hypothermia associated with intravenous t-PA is in progress (ICTuS 2/3). This design, however, has problems, since the neuroprotectant used might reduce the rate of recanalization by interfering with intravenous $\mathrm{t}$-PA, or be detrimental when early reperfusion does not occur ( $60 \%$ of patients treated with intravenous t-PA).

In conclusion, the available data suggest that although intravenous t-PA is not beneficial beyond $4.5 \mathrm{~h}$ if patient selection is based on plain CT only, it might be beneficial up to $6 \mathrm{~h}$ in the target mismatch profile. Endovascular treatment, either as a bridging therapy or in isolation, may prove beneficial up to and perhaps even beyond $12 \mathrm{~h}$ in highly selected patients, who are probably not so uncommon. This will need to be documented using stateof-the-art trials. Finally, future trials of neuroprotection should combine the agent with reperfusion therapy. Although agents targeting nonneuronal targets such as the neurovascular unit and inflammation could be started in the acute stage, admittedly even before reperfusion therapy, clinical evidence supporting their usefulness is still lacking.

\section{Acknowledgments}

The author is grateful to G. Albers, M. Lansberg, S. Christiansen, M. Parsons, A. Bivard and A. Alexandrov for helpful discussions and unpublished data.

\section{References}

$>1$ Baron JC, Bousser MG, Comar D, Soussaline F, Castaigne P: Noninvasive tomographic study of cerebral blood flow and oxygen metabolism in vivo. Potentials, limitations, and clinical applications in cerebral ischemic disorders. Eur Neurol 1981;20:273-284.

-2 Wise RJ, Bernardi S, Frackowiak RS, Legg NJ, Jones T: Serial observations on the pathophysiology of acute stroke. The transition from ischaemia to infarction as reflected in regional oxygen extraction. Brain 1983;106: 197-222.

$>3$ Hakim AM, Pokrupa RP, Villanueva J, Diksic M, Evans AC, Thompson CJ, Meyer E, Yamamoto YL, Feindel WH: The effect of spontaneous reperfusion on metabolic function in early human cerebral infarcts. Ann Neurol 1987;21:279-289.

4 Heiss WD, Huber M, Fink GR, Herholz K, Pietrzyk U, Wagner R, Wienhard K: Progressive derangement of periinfarct viable tissue in ischemic stroke. J Cereb Blood Flow Metab 1992;12:193-203.

5 Greenberg JP, Correia JA, Alpert NM, Buonanno FS: Distribution and incidence of tissue with elevated oxygen extraction fraction in acute ischemic stroke. J Cereb Blood Flow Metab 1993;13:S599.

6 Marchal G, Serrati C, Rioux P, Petit-Taboue MC, Viader F, de la Sayette V, Le Doze F, Lochon P, Derlon JM, Orgogozo JM, et al: PET imaging of cerebral perfusion and oxygen consumption in acute ischaemic stroke: relation to outcome. Lancet 1993;341:925-927.

7 Marchal G, Rioux P, Serrati C, Furlan M, Derlon JM, Viader F, Baron JC: Value of acute-stage positron emission tomography in predicting neurological outcome after ischemic stroke: further assessment. Stroke 1995; 26:524-525.

8 Marchal G, Beaudouin V, Rioux P, de la Sayette V, Le Doze F, Viader F, Derlon JM, Baron JC: Prolonged persistence of substantial volumes of potentially viable brain tissue after stroke: a correlative PET-CT study with voxel-based data analysis. Stroke 1996;27:599-606.

>9 Marchal G, Furlan M, Beaudouin V, Rioux P, Hauttement JL, Serrati C, de la Sayette V, Le Doze F, Viader F, Derlon JM, Baron JC: Early spontaneous hyperperfusion after stroke. A marker of favourable tissue outcome? Brain 1996;119:409-419.

10 Jones TH, Morawetz RB, Crowell RM, Marcoux FW, FitzGibbon SJ, DeGirolami U, Ojemann RG: Thresholds of focal cerebral ischemia in awake monkeys. J Neurosurg 1981;54:773-782. 
11 Darby DG, Barber PA, Gerraty RP, Desmond PM, Yang Q, Parsons M, Li T, Tress BM, Davis SM: Pathophysiological topography of acute ischemia by combined diffusion-weighted and perfusion MRI. Stroke 1999; 30:2043-2052.

12 Davis SM, Donnan GA, Parsons MW, Levi C, Butcher KS, Peeters A, Barber PA, Bladin C, De Silva DA, Byrnes G, Chalk JB, Fink JN, Kimber TE, Schultz D, Hand PJ, Frayne J, Hankey G, Muir K, Gerraty R, Tress BM, Desmond PM: Effects of alteplase beyond $3 \mathrm{~h}$ after stroke in the echoplanar imaging thrombolytic evaluation trial (EPITHET): a placebo-controlled randomised trial. Lancet Neurol 2008;7:299-309.

13 Albers GW, Thijs VN, Wechsler L, Kemp S, Schlaug G, Skalabrin E, Bammer R, Kakuda W, Lansberg MG, Shuaib A, Coplin W, Hamilton S, Moseley M, Marks MP: Magnetic resonance imaging profiles predict clinical response to early reperfusion: the diffusion and perfusion imaging evaluation for understanding stroke evolution (DEFUSE) study. Ann Neurol 2006;60:508-517.

14 Lansberg MG, Lee J, Christensen S, Straka M, De Silva DA, Mlynash M, Campbell BC, Bammer R, Olivot JM, Desmond P, Davis SM, Donnan GA, Albers GW: Rapid automated patient selection for reperfusion therapy: a pooled analysis of the echoplanar imaging thrombolytic evaluation trial (EPITHET) and the diffusion and perfusion imaging evaluation for understanding stroke evolution (DEFUSE) study. Stroke 2011;42:16081614.

15 Lansberg MG, Straka M, Kemp S, Mlynash M, Wechsler LR, Jovin TG, Wilder MJ, Lutsep HL, Czartoski TJ, Bernstein RA, Chang CW, Warach S, Fazekas F, Inoue M, Tipirneni A, Hamilton SA, Zaharchuk G, Marks MP, Bammer R, Albers GW: MRI profile and response to endovascular reperfusion after stroke (DEFUSE 2): a prospective cohort study. Lancet Neurol 2012;11:860-867.

16 Fieschi C, Argentino C, Lenzi GL, Sacchetti ML, Toni D, Bozzao L: Clinical and instrumental evaluation of patients with ischemic stroke within six hours. J Neurol Sci 1989;91:311-321.

17 Saqqur M, Tsivgoulis G, Molina CA, Demchuk AM, Garami Z, Barreto A, Spengos K, Forteza A, Mikulik R, Sharma VK, Brunser A, Martinez P, Montaner J, Kohrmann M, Schellinger PD, Alexandrov AV: Design of a PROspective multi-national CLOTBUST collaboration on reperfusion therapies for stroke (CLOTBUST-PRO). Int J Stroke 2008;3:66-72.

18 Furlan A, Higashida R, Wechsler L, Gent M, Rowley H, Kase C, Pessin M, Ahuja A, Callahan F, Clark WM, Silver F, Rivera F: Intra-arterial prourokinase for acute ischemic stroke. The PROACT II study: a randomized controlled trial. Prolyse in Acute Cerebral Thromboembolism. JAMA 1999;282:2003-2011.

19 Hennerici MG, Kern R, Szabo K: Non-pharmacological strategies for the treatment of acute ischaemic stroke. Lancet Neurol 2013;12:572-584.

20 Khatri P, Abruzzo T, Yeatts SD, Nichols C, Broderick JP, Tomsick TA: Good clinical outcome after ischemic stroke with successful revascularization is time-dependent. Neurology 2009;73:1066-1072.

-21 Jovin TG, Liebeskind DS, Gupta R, Rymer M, Rai A, Zaidat OO, AbouChebl A, Baxter B, Levy EI, Barreto A, Nogueira RG: Imaging-based endovascular therapy for acute ischemic stroke due to proximal intracranial anterior circulation occlusion treated beyond $8 \mathrm{~h}$ from time last seen well: retrospective multicenter analysis of 237 consecutive patients. Stroke 2011;42:2206-2211.

22 Chavez JC, Hurko O, Barone FC, Feuerstein GZ: Pharmacologic interventions for stroke: looking beyond the thrombolysis time window into the penumbra with biomarkers, not a stopwatch. Stroke 2009;40:e558e563.

23 Minnerup J, Sutherland BA, Buchan AM, Kleinschnitz C: Neuroprotection for stroke: current status and future perspectives. Int J Mol Sci 2012; 13:11753-11772.

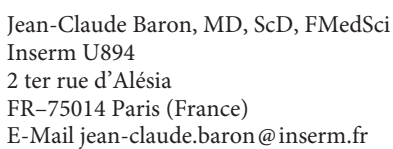

\section{Assessment of Outcome in Stroke Trials: In Rodents}

\section{U. Dirnagl}

Center for Stroke Research Berlin, Charité Universitätsmedizin Berlin, and German Center for Neurodegeneration Research, Berlin, Germany

At present, the only pharmacological treatment of acute ischemic stroke with proven efficacy is thrombolysis with recombinant tissue plasminogen activator. Worldwide stroke researchers strive to develop neuroprotective and neuroregenerative treatments to alleviate the tremendous burden of this disease on patients, relatives and societies. Effectiveness of a treatment is measured as an improvement of outcome. Clinical stroke trials use endpoints such as improvement in function in daily living, reduction in mortality, or recurrence of stroke or other cardiovascular events [see companion article by Adams Jr., pp. 318]. Surrogate endpoints, such as infarct volume, or biomarkers are used for hypothesis generation and to obtain proof of concept for a biological effect of a treatment, but they are not relevant for approval of a drug by the Food and Drug Administration or European Medical Agencies. In preclinical stroke research, however, reductions in infarct volume have been the gold standard to determine efficacy of an experimental treatment for decades. Functional outcome, on the other hand, has played a minor role and only entered the field with the need to evaluate putative neurorestorative treatments. In the following, I will briefly discuss how stroke is modeled in rodents [for more details on treatment, see companion article by Macrae, pp. 308], and consequently how outcome is evaluated based on infarct volume measurements or assessment of function. Particular attention will be placed on the pitfalls of such measurements and on other relevant limitations of results of preclinical stroke research based on assessing outcome in experimental models of stroke.

\section{Modeling of Stroke in Rodents}

Preclinical stroke research is dominated by modeling of middle cerebral artery (MCA) occlusion (MCAO) in rodents, in particular in the mouse and rat. MCAO can be achieved without craniotomy by intravascular advancement of an occluding plastic filament via the common carotid artery ('proximal' MCAO, i.e. affecting the vascular supply of the striatum and neocortex) or by cauterization or ligature of the MCA via a surgical transtemporal approach ('distal' MCAO, i.e. not affecting the lenticulostriate arteries and hence purely neocortical ischemia). 
Thrombotic MCAO can be achieved by placing preformed thrombi in the cerebral circulation via a catheter, intracranial intra-arterial thrombin injection or in situ thrombus formation via a photoactivator (such as rose bengal) and laser illumination of the vessel. Some of these approaches allow transient MCAO (e.g. by retraction of the occluding filament), and in some occlusion must remain permanent. The common characteristic of all these models, but also its limitation, is the fact that they require anesthesia of variable duration (from 5 to $45 \mathrm{~min}$ ) and that they attempt to mimic large strokes within the MCA territory.

\section{Infarct Volume Determination}

The most widely used outcome measure of experimental stroke studies in rodents is the measurement of infarct size (usually as a volume). Infarct size is determined either histologically via serial sectioning and staining after brain extraction, histochemically by incubating fresh unprocessed brain slices with chemicals that produce dyes from colorless substrates when exposed to mitochondrial enzymes, e.g. 2,3,5-triphenyltetrazolium chloride (TTC), or MRI [for extensive reviews, see ref. 1,2]. Pitfalls of these techniques include the need for edema correction, the fact that, depending on the model, damage may evolve over many hours and even days, while 'infarct volumes' start to apparently decrease after several days again due to extensive remodeling of the tissue and absorption of dead tissue. Invasion of inflammatory cells after a few days may mask the lesion by converting indicators for viable tissue in histochemical infarct determination, such as the one using TTC. Many experimental stroke studies measure infarct volume at $24 \mathrm{~h}$, when infarcts have may not have fully matured and edema is still prominent. A 'neuroprotectant' found effective in such studies may have only delayed damage or decreased brain edema. Studies measuring outcome via infarct volumes beyond 3 days may be confounded by effects of the putative 'neuroprotectant' on tissue remodeling or invasion of inflammatory cells. Hence, results of outcome studies in the rodent are highly dependent on the time interval between MCAO and infarct volume determination, and may be confounded by numerous factors.

\section{Assessment of Functional Outcome in Rodents}

Rodents cannot communicate the level of their impairment after MCAO. Functional outcome measurements therefore depend on a plethora of tests, many of which require training before MCAO and highly skilled personnel, and are very time consuming. Consequently, many investigators prefer simple but rather insensitive tests (such as the Bederson score: $0=$ normal; $1=$ forelimb flexion; 2 = decreased resistance to lateral push, and $3=$ circling), rota-rod or sticky tape removal, all of which are highly based on sensorimotor function. MCAO may affect somatosensory or motor function, memory or executive function, or cause depression. For each affected function, specific tests exist [for an extensive treatment of functional testing after stroke see ref. 3 (in the mouse) and 4 (in the rat)]. A major problem of functional testing in rodents is their exquisite capacity to compensate the deficit, either by using alternative strategies while performing the test (often hard to detect by the investigator) or by brain plasticity. In any case, many of the functional deficits which clearly demonstrate pathology a few days after MCAO return to normal and are not distinguishable from sham controls within a few days. In combination with a rather high variance in test results, it is exceedingly difficult to demonstrate a deficit in many MCAO models after more than 1 week, let alone the protective effect of a compound when compared to untreated controls. Because of the low sensitivity of the tests, many investigators use batteries of tests, some of which produce a multitude of quantitative scores and variables. For example, a popular semiautomated system to score motor function (cat walk) includes $>50$ variables per test run. As a result, many studies compare a multitude of variables between experimental groups, often without correcting for multiple testing and sometimes possibly post hoc 'cherry picking' of statistically significant variables.

\section{Internal Validity}

To interpret outcome measures reported by experimental stroke studies it is important to be aware of the fact that stroke research as well as preclinical modeling in biomedicine in general may be confounded by issues of internal validity. In particular, many preclinical stroke studies do not randomize, group allocation is transparent throughout the experiment, and outcome is not assessed in a blinded fashion. In other words, methods to prevent bias, which are standard practice in clinical medicine, are not yet widely implemented in preclinical medicine. This may lead to an overestimation of effect size and false-positive results. In addition, most preclinical stroke studies use group sizes of 5-10, which often results in a statistical power of around 0.5 and very low positive 
Fig. 1. Graphical representation of infarct volume reduction of a typical experimental stroke study (data simulated). $\mathrm{n}=8$ per group. ${ }^{*} \mathrm{p}<0.05$. Illusion of robust reduction in infarct volume using bar graphs and SEM (a) and representation of individual data points (dot plot) and box and whisker graph (b) demonstrating true variance of the data and substantial overlap of the groups. c After exclusion of one data point (crossed out in d), bar graph appears largely unchanged, but statistical significance is lost $(\mathrm{p}=0.1)$. $\mathbf{d}$ Exclusion of a single data point changes the interpretation of the data. Please note that this is of particular relevance with small group sizes and in the absence of predefined inclusion and exclusion criteria, as well as nonblinded assessment of outcome (as in most preclinical stroke studies).

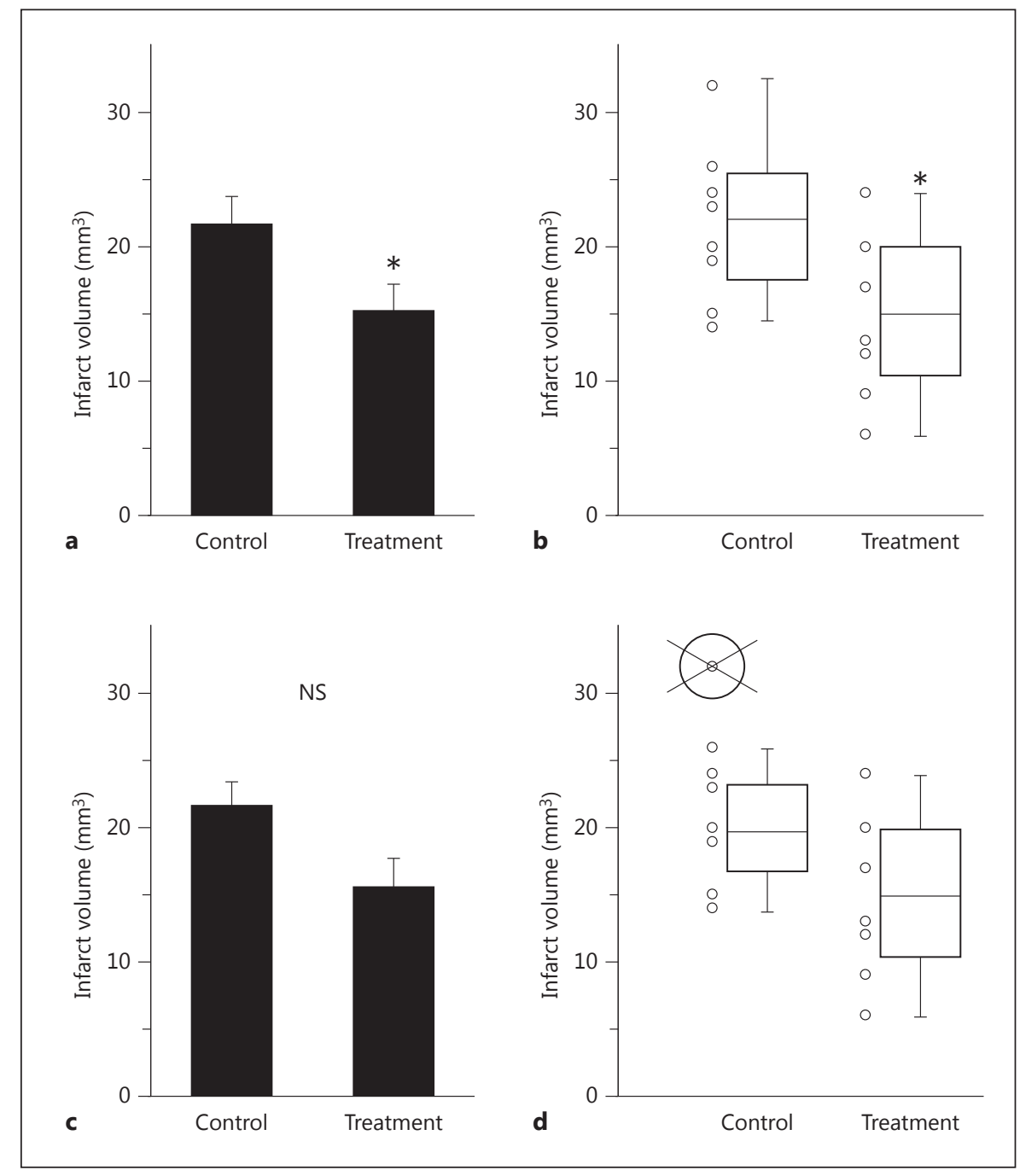

predictive values $[5,6]$. Figure 1 demonstrates how sensitive such experimental designs are to the alteration of just one value.

\section{External Validity}

When interpreting promising results (decrease in infarct volume or improvement in function) of putative neuroprotectants in experimental stroke studies, and in particular if such results suggest their possible validation in a clinical trial, external validity of the experiment becomes highly relevant. Most preclinical stroke studies use healthy, male, inbred, adolescent rodents raised under specific pathogen-free conditions. This is equivalent to clinical testing in healthy, briefly anesthetized pubertal male twins raised in $6-\mathrm{m}^{2}$ isolator tents on an enriched granola diet. Needless to say that stroke patients are of both sexes, usually elderly and comorbid, receive multiple medications, and have been exposed to multiple pathogens and antigens throughout life. Fortunately, many of these factors can be modeled in the rodent as well, but due to the extra costs and complexities of such experiments, this is rarely done. In most instances where it has been done, relevant differences with respect to the efficacy of putative neuroprotectants between the young healthy and the aged comorbid animals became apparent. 


\section{Conclusions}

Outcome in preclinical stroke studies is often measured in the short term (1-3 days), with a strong focus on reductions in infarct volume. Numerous tests exist to assess functional outcome after stroke in rodents, but they are labor intensive and often quite insensitive, in particular due to the very high capacity of rodents for spontaneous recovery. A number of pitfalls exist which may affect the interpretation of these outcome measurements. Taken together, assessment of outcome in preclinical and clinical stroke trials is quite different, hampering the translation of results between both realms. In addition, deficiencies in internal and external validity, as well as the reporting of preclinical stroke studies, may affect their robustness and relevance for bench-to-bedside translation. However, a concerted effort of preclinical and clinical stroke researchers may overcome these shortcomings. The following recommendations reflect the current discussion in the field:

Preclinical stroke studies should conform to the ARRIVE (Animal Research: Reporting in vivo Experiments) guidelines when reporting animal experiments [7].

- Preclinical stroke studies should prespecify and report inclusion and exclusion criteria $[5,8]$.

- Bias should be minimized by randomization, allocation concealment and blinding $[5,8]$.

- External validity should be improved by modeling of confounders such as age, gender and comorbidities, in particular when approaching clinical trials.

- Significance of results in preclinical trials should be increased by increasing group size [9].

- Critical findings need to be replicated [9].

\section{Acknowledgments}

U.D. is supported by the European Union's Seventh Framework Program (FP7/2008-2013) under Grant Agreements 201024 and 202213 (European Stroke Network); the Deutsche Forschungsgemeinschaft (NeuroCure Cluster of Excellence, Exc 257) and the Bundesministerium für Bildung und Forschung (Center for Stroke Research Berlin, 01 EO 08 01).

\section{References}

1 Zille M, Farr TD, Przesdzing I, Müller J, Sommer C, Dirnagl U, Wunder A: Visualizing cell death in experimental focal cerebral ischemia: promises, problems, and perspectives. J Cereb Blood Flow Metab 201;32:213231.

-2 Farr TD, Wegener S: Use of magnetic resonance imaging to predict outcome after stroke: a review of experimental and clinical evidence. J Cereb Blood Flow Metab 2010;30:703-717.
3 Balkaya M, Kröber JM, Rex A, Endres M: Assessing post-stroke behavior in mouse models of focal ischemia. J Cereb Blood Flow Metab 2013;33: 330-338.

4 Metz GA: Behavioral testing in rodent models of stroke; in Dirnagl U (ed): Rodent Models of Stroke. Neuromethods. New York, Springer, 2010, vol 47, pp 199-212.

5 Dirnagl U: Bench to bedside: the quest for quality in experimental stroke research. J Cereb Blood Flow Metab 2006;26:1465-1478.

6 Button KS, Ioannidis JP, Mokrysz C, Nosek BA, Flint J, Robinson ES, Munafò MR: Power failure: why small sample size undermines the reliability of neuroscience. Nat Rev Neurosci 2013;14:365-376.

-7 Kilkenny C, Browne W, Cuthill IC, Emerson M, Altman DG, National Centre for the Replacement, Refinement and Reduction of Animals in Research: Animal research: reporting in vivo experiments - the ARRIVE guidelines. J Cereb Blood Flow Metab 2011;31:991-993.

$\rightarrow 8$ Landis SC, Amara SG, Asadullah K, Austin CP, Blumenstein R, Bradley EW, Crystal RG, Darnell RB, Ferrante RJ, Fillit H, Finkelstein R, Fisher M, Gendelman HE, Golub RM, Goudreau JL, Gross RA, Gubitz AK, Hesterlee SE, Howells DW, Huguenard J, Kelner K, Koroshetz W, Krainc D, Lazic SE, Levine MS, Macleod MR, McCall JM, Moxley RT 3rd, Narasimhan K, Noble LJ, Perrin S, Porter JD, Steward O, Unger E, Utz U, Silberberg SD: A call for transparent reporting to optimize the predictive value of preclinical research. Nature 2012;490:187-191.

$\checkmark 9$ Dirnagl U, Hakim A, Macleod M, Fisher M, Howells D, Alan SM, Steinberg G, Planas A, Boltze J, Savitz S, Iadecola C, Meairs S: A concerted appeal for international cooperation in preclinical stroke research. Stroke 2013;44:1754-1760.

Prof. Dr. Ulrich Dirnagl

Department of Neurology and Experimental Neurology and

Center for Stroke Research Berlin

Charité Universitätsmedizin Berlin

Charitéplatz 1

DE-10117 Berlin (Germany)

E-Mail ulrich.dirnagl@ charite.de

\section{Clinical Trials in Stroke: Issues in Design and Use of Clinical Rating Instruments}

Harold P. Adams Jr.

Division of Cerebrovascular Diseases, Department of Neurology, Carver College of Medicine, Comprehensive Stroke Center, University of lowa Hospitals and Clinics, University of lowa, lowa City, lowa, USA

The ultimate goal of experimental and clinical research in stroke is to prevent or lessen the consequences of the brain injury in humans. Preventing, treating or augmenting recovery in patients is the real measure of success in stroke research. Unfortunately, the translation of scientific success in the laboratory to patient care has been difficult. At present, the primary success in treatment of acute stroke has been intravenous thrombolysis. Other promising therapies have not been successful when tested in a patient care setting. Interventions that are effective in animal models often have not worked in humans. The lack of success is a source of considerable frustration among both experimental and clinical re- 
searchers [1-3]. This contribution, which is from the view of a clinical researcher, highlights some of the differences between experimental and clinical stroke research that may partially explain the painful transition from experimental to clinical research. The goal is to expand the dialogue among all stroke researchers, which in turn could hopefully ease the translation of advances in the experimental setting to the treatment of patients with cerebrovascular disease.

\section{Issues in the Design of Clinical Trials in Stroke}

A clinical trial is the culmination of a series of experimental and human research projects that are very expensive and arduous with the series of studies often spanning a decade or longer. Because of the considerable resources required to test promising therapies, a limited number of interventions can be tested. Still, the results of the phase III trials become the foundation for advances in patient care because their data are used by the panels that write guidelines (evidence-based medicine) [4]. In addition, the results of the phase III trials are applied by regulatory bodies to approve new therapies in the management of patients with cerebrovascular disease.

The construction of the research plan for a clinical trial differs considerably from a laboratory experiment and requires considerable thought. The basic goal is to recruit, treat and assess an adequate number of subjects to accurately test the clinical research question in as short a time as possible. Often, relatively large numbers of subjects are needed to detect a subtle but important difference in outcomes among the treatment groups and as a consequence most trials involve the collaboration of multiple institutions often on an international scale.

There are two general strategies for the design of a clinical trial. One option is to enroll a carefully defined subgroup of patients by the use of restrictive entry criteria [5, 6]. Entry may be influenced by specific clinical features or the results of ancillary diagnostic studies, such as brain imaging, which means that stringent quality control measures need to be in place to assure that all the components of the assessments are done with accuracy. A focused trial may use modality-specific outcome measurements supplemented by global outcome scales. While these trials may need to recruit a small number of subjects, recruitment may be difficult because of the strict entry criteria. In addition, the results of such a trial will be applicable to only a limited number of patients, which may restrict its clinical impact. Another option is to enroll a diverse group of subjects with stroke using broad entry criteria and to employ standardized global outcome scales that allow comparison of results with other clinical trials. An advantage of this approach is that the results of the trial generally are applicable to a wider population of patients with stroke. The standard (usual) components of the trial include measures to prevent bias, plans for ongoing monitoring of the data for quality and prespecified plans for analysis of the data. The usual measures to prevent bias are randomization to assure that the baseline characteristics of the study groups are similar, and blinding (masking) of subjects and investigators to study intervention assignment to assure that reporting of adverse and outcome events is not influenced by knowledge of the treatment group. In circumstances in which blinding (masking) of the subjects and investigators is not possible (for example a trial comparing a surgical intervention with usual medical therapy), central blinded adjudication of outcome events may be performed. Stratification of subjects, most commonly by baseline severity of stroke, age or trial site, is often included in the design. Most trials are designed using the intention-to-treat principle, which means that all events are counted in the originally assigned treatment arm regardless of actual treatment [7]. In this design, the investigators must avoid crossovers. Because of the large size of the trials, aggressive recruitment plans need to be in place. In addition, the duration of follow-up for patients in a stroke trial is usually 3-12 months to determine if the tested therapy has made an impact on outcome. As a result, measures to assure retention of subjects until the planned complication of the trial are equally critical. The latter issue is particularly vexing because subjects have the right to withdraw their participation or they may become lost to follow-up.

Several factors, which may affect prognosis or response to treatment, add to the complexity of the design of a clinical trial in stroke. Stroke results from a broad spectrum of vascular diseases. The locations and sizes of the brain injuries vary considerably, which lead to a wide range of neurological impairments of differing severity. Epidemiological factors such as the subject's age, sex and ethnicity may also affect responses to treatment. The subjects' preexisting health and the presence of serious comorbid diseases also affect prognosis. In addition, most stroke patients require multiple therapies, including medications to prevent recurrent stroke or to treat neurological or medical complications, rehabilitation and, on occasion, surgical interventions. The many variables also lead to the necessity of recruiting a large number of subjects, which in turn leads to the creation of complex mul- 
ticenter or multinational collaborative studies that involve a large number of local investigators and supporting personnel.

The primary measure of success of an intervention in stroke is the patient's overall (favorable) outcome with the goal of determining if the tested therapy reduces the likelihood of severe neurological impairments, disability or handicap. Generally, mortality is not used as a primary outcome because severely incapacitated survivorship may be worse than death, and a trial does not want to demonstrate that a therapy reduces mortality at the cost of more severely disabled survivors. There is considerable research in the utility of changes in brain and vascular imaging findings as measures of success using smaller volumes of infarction or early recanalization. Some correlation with clinical outcomes is present but these ancillary studies have not supplanted clinical outcomes. In addition, biomarkers are being assessed for the selection of patients to treat or for determining outcome after stroke. As of yet, the results of these tests are not a substitute for clinical outcomes.

\section{Stroke Scales}

Stroke scales provide important information and ease communication; they are a critical component of clinical research. Scales may be used to rate the severity and type of neurological impairments, to determine eligibility for enrollment, to forecast outcomes, to monitor changes in the neurological status, to examine responses to treatment and to determine outcome after stroke. A useful clinical stroke scale must have inherent credibility and face validity. It should be easy to administer and to score. The scale should be tested for reliability, interrater agreement and intrarater reproducibility. Researchers have an extra burden to assure that the scale is administered correctly and that the scoring is accurate and consistent. Modern trials include educational and certification programs to assure that the raters are using the scale in a correct manner. In addition, central adjudication of outcome can also be performed. In summary, the results of the scale should be believable and make sense for both health care providers and the public. A knowledgeable person should have a mental image of the patient's condition when the score on a stroke scale is assigned.

A combination of rating instruments may be used for different components of a clinical trial. In general, scales of neurological impairment are used to establish eligibility for enrollment and monitor responses to treatment including safety and to determine outcome at the end of the trial. The score on a scale of neurological impairment often affects the selection of subjects in the trial by excluding those with very mild or severe deficits. The score can also be used as a stratification factor to assure similarities in the treatment arms. The NIH Stroke Scale is the most commonly used scale of neurological impairment [8]. It contains 15 items assessed by neurological examination and it can be performed rapidly by a wide range of professionals. It is a well-validated quantitative measure of neurological impairments that is highly predictive of outcome. It has high interrater agreement and reproducibility. It is adapted to multiple languages and cultures. Educational and certification programs are available. It has also been employed in general patient care.

Global scales of recovery after stroke are widely accepted by the medical community, funding agencies and government regulators. These scales broadly differentiate outcomes that reflect the impact of multiple neurological impairments and they are used in both acute treatment and recovery trials. The global scales may miss some important improvements such as discrete areas of neurological disability because they overemphasize some components of recovery. They may also have ceiling and floor effects. The modified Rankin scale is an internationally accepted global outcome scale that has scores ranging from 0 (normal) to 6 (dead) [9]. Each score has its own definition, and an emphasis of the scale involves motor limitations and walking. The results of the scores in the subject populations are analyzed into favorable or unfavorable outcomes using a variety of statistical methods (dichotomous, adjusted dichotomous based on initial impairments, trichotomous or distributional).

Modality-specific scales examine responses to an intervention aimed at a specific impairment or disability. As such, these scales are often used in rehabilitation or recovery research. An example is the Fugl-Meyer scale for assessing motor recovery after stroke [10]. An advantage of these instruments is that different impairments may resolve at differing rates. In general, these scales do not provide data on overall outcome. The scores of the scales are not understood by clinicians or the public, and the data from these scales may not be easy to compare with data recorded in other clinical trials.

Increasingly, quality-of-life scales are being included in stroke research trials. These rating instruments cover a broad range of functioning, including physical, psychological and general health. They are also affected by the subject's social situation and influenced by the person's experiences, beliefs, expectations and perceptions. The 
most commonly used scales are the EuroQoL and the Stroke Impact Scale. In general, the quality of life measures have not been the primary way to measure success of treatment of an acute stroke.

\section{Conclusions}

Experimental research remains crucial for advancing our understanding about stroke and the brain's ability to recover. Derived information is fundamental for testing promising therapies and the results of laboratory studies greatly influence clinical research. However, the issues addressed in clinical research differ greatly from experimental science. It is difficult or impossible for the clinical researcher to replicate laboratory experiments. Basic science researchers in stroke should consider the difficulties inherent in the clinical setting. These insights are needed to ease the translation of knowledge from the laboratory to the patient.

\section{References}

-1 Dirnagl U, Fisher M: REPRINT: international, multicenter randomized preclinical trials in translational stroke research: it is time to act. Stroke 2012;43:1453-1454.

2 Fisher M: New approaches to neuroprotective drug development. Stroke 2011;42(1 suppl):S24-S27.

3 del Zoppo GJ: Clinical trials in acute stroke: why have they not been successful? Neurology 1998;51(3 suppl 3):S59-S61.

4 Jauch EC, Saver JL, Adams HP Jr, et al: Guidelines for the early management of patients with acute ischemic stroke: a guideline for healthcare professionals from the American Heart Association/American Stroke Association. Stroke 2013;44:870-947.

5 Fisher M, Albers GW: Advanced imaging to extend the therapeutic time window of acute ischemic stroke. Ann Neurol 2013;73:4-9.

6 Lansberg MG, Thijs VN, Bammer R, et al: The MRA-DWI mismatch identifies patients with stroke who are likely to benefit from reperfusion. Stroke 2008;39:2491-2496.

7 Bath PMW, Lees KR, Schellinger PD, et al: Statistical analysis of the primary outcome in acute stroke trials. Stroke 2012;43:1171-1178.

$>8$ Brott T, Adams HP Jr, Olinger CP, et al: Measurements of acute cerebral infarction: a clinical examination scale. Stroke 1989;20:864-870.

$\checkmark 9$ van Swieten JC, Koudstaal PJ, Visser MC, Schouten HJ, van Gijn J: Interobserver agreement for the assessment of handicap in stroke patients. Stroke 1988;19:604-607.

10 Duncan PW, Propst M, Nelson SG: Reliability of the Fugl-Meyer assessment of sensorimotor recovery following cerebrovascular accident. Phys Ther 1983;63:1606-1610.

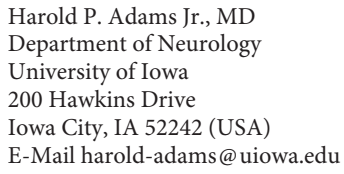

